\title{
High-Order Contamination in the Tail of Gravitational Collapse
}

\author{
Shahar Hod \\ The Racah Institute for Physics, The Hebrew University, Jerusalem 91904, Israel
}

(August 11, 2018)

\begin{abstract}
It is well known that the late-time behaviour of gravitational collapse is dominated by an inverse power-law decaying tail. We calculate higher-order corrections to this power-law behaviour in a spherically symmetric gravitational collapse. The dominant "contamination" is shown to die off at late times as $M^{2} t^{-4} \ln (t / M)$. This decay rate is much slower than has been considered so far. It implies, for instance, that an 'exact' (numerical) determination of the power index to within $\sim 1 \%$ requires extremely long integration times of order $10^{4} M$. We show that the leading order fingerprint of the black-hole electric charge is of order $Q^{2} t^{-4}$.
\end{abstract}

\section{INTRODUCTION}

Waves propagating in a curved spacetime develop "tails". In particular, it is well established that the dominant late-time behaviour of massless fields propagating in black-hole spacetimes is a power-law tail. Price [1] was the first to analyze the mechanism by which the spacetime outside a (nearly spherical) collapsing star divests itself of all radiative multipole moments, and leaves behind a Schwarzschild black hole; it was demonstrated that all radiative perturbations decay asymptotically as an inverse power of time. Physically, these inverse power-law tails are associated with the backscattering of waves off the effective curvature potential at asymptotically far regions [2,1]. 
The analysis of Price was extended by other authors. Bičák [3] generalized the analysis and studied the dynamics of a scalar field in a charged Reissner-Nordström spacetime. He also found an asymptotic inverse power-law decay of the field, with the same power indices as in the Schwarzschild spacetime (with the exception of the extremal Reissner-Nordström black hole [3]). In a brilliant work, Leaver [4] demonstrated that the late-time tail can be associated with the existence of a branch cut in the Green's function for the wave propagation problem.

Gundlach, Price, and Pullin [5] showed that these inverse power-law tails also characterize the late-time evolution of radiative fields at future null infinity, and at the black-hole outer horizon. Furthermore, they showed that power-law tails are a genuine feature of gravitational collapse - the existence of these tails was demonstrated in full non-linear numerical simulations of the spherically symmetric collapse of a self-gravitating scalar field [6] (this was later reproduced in [7]).

Our current understanding of the late-time tail is, however, somewhat unsatisfactory. The (leading order) power-law tails in black-hole spacetimes are well established [1 [10], but the resultant formulae are only truly useful at very late times. In a typical evolution scenario there is a considerable time window in which the signal is no longer dominated by the quasinormal modes [4, 11, 5, 6, 12, 8, 9], but the leading order power-law tail has not yet taken over [9].

The purpose of this paper is to derive analytic expressions for the higher-order corrections which "contaminate" the well-known power-law tail in a spherically symmetric gravitational collapse. The determination of these higher-order terms is important from several points of view: The analyses of Bičák [3] and Gundlach et. al. [5] established the fact that the leading-order power-law tail is universal in the sense that it is independent of the black-hole electric charge (i.e., the power index in a charged Reissner-Nordström spacetime was shown to be identical with the one found by Price [1] for the neutral Schwarzschild black hole). This observation begs the question: what fingerprints (if any) does the black-hole electric charge leave on the field's decay ? 
Moreover, the calculation of higher-order corrections to the leading order power-law tail is also of practical importance; this is especially crucial for the determination of the power index from numerical simulations. The dominant inverse power-law tail is "contaminated" by higher-order terms, whose effect become larger as the aveliable time of integration decreases. The precise power index is expected only at infinitely-late time. Thus, in practice, the limited time of integration introduces an inherent error in the determination of the power index. The only systematic approach to quantify the errors which are introduced by the finite integration time is to study higher-order corrections. If one computes the contaminated part of the late-time tail, then the ratio of the corrections to the leading order term is a systematic, quantitative, indication of the error caused by the finite-time numerical calculation.

These questions and several others are addressed in the present paper. The plan of the paper is as follows. In Sec. [1] we give a short description of the physical system and formulate the evolution equation considered. In Sec. [II] we give an analytical description of the late-time evolution of scalar fields in black-hole spacetimes. In Sec. IV we confirm our analytical results by numerical simulations. We conclude in Sec. $\nabla$ with a brief summary of our results and their implications.

\section{DESCRIPTION OF THE SYSTEM}

We consider the evolution of a spherically symmetric massless scalar field in a spherically symmetric charged background (a collapsing star or a fixed black hole). The external gravitational field of a spherically symmetric charged object of mass $M$ and charge $Q$ is given by the Reissner-Nordström metric

$$
d s^{2}=-\left(1-\frac{2 M}{r}+\frac{Q^{2}}{r^{2}}\right) d t^{2}+\left(1-\frac{2 M}{r}+\frac{Q^{2}}{r^{2}}\right)^{-1} d r^{2}+r^{2} d \Omega^{2} .
$$

Using the tortoise radial coordinate $y$, which is defined by $d y=d r /\left(1-2 M / r+Q^{2} / r^{2}\right)$, the line element becomes

$$
d s^{2}=\left(1-\frac{2 M}{r}+\frac{Q^{2}}{r^{2}}\right)\left(-d t^{2}+d y^{2}\right)+r^{2} d \Omega^{2},
$$


where $r=r(y)$.

The wave equation $\phi_{; a b} g^{a b} \phi=0$ for the scalar field in the black-hole background is

$$
\psi_{, t t}-\psi_{, y y}+V \psi=0
$$

where

$$
V=V_{M, Q}(r)=\left(1-\frac{2 M}{r}+\frac{Q^{2}}{r^{2}}\right)\left(\frac{2 M}{r^{3}}-\frac{2 Q^{2}}{r^{4}}\right) .
$$

In terms of the tortoise coordinate $y$ and for $y \gg M$ the curvature potential Eq. (四) reads

$$
V=V(y)=\frac{2 M}{y^{3}}+\frac{12 M^{2} \ln y}{y^{4}}-\frac{4 M^{2}+2 Q^{2}}{y^{4}}+O\left(\frac{M^{3} \ln y}{y^{5}}\right) \text {. }
$$

\section{THE LATE-TIME BEHAVIOUR}

The general solution to the wave-equation (3) can be written as a series depending on two arbitrary functions $F$ and $G$ [1]

$$
\begin{aligned}
\psi= & G^{(0)}(u)+F^{(0)}(v) \\
& +\sum_{k=0}^{\infty} B_{k}(y)\left[G^{(-k-1)}(u)+(-1)^{k} F^{(-k-1)}(v)\right] .
\end{aligned}
$$

Here $u \equiv t-y$ is a retarded time coordinate and $v \equiv t+y$ is an advanced time coordinate.

For any function $H, H^{(k)}$ is the $k$ th derivative of $H^{(0)}$; negative-order derivatives are to be interpreted as integrals. The first two terms in Eq. (6) represent the zeroth-order solution (with $V=0$ ).

The star begins to collapse at a retarded time $u=u_{0}$. The world line of the stellar surface is asymptotic to an ingoing null line $v=v_{0}$, while the variation of the field on the stellar surface is asymptotically infinitely redshifted [1, 3]. This effect is caused by the time dilation between static frames and infalling frames. A static external observer sees all processes on the stellar surface become "frozen" as the star approaches the horizon. Thus, he sees all physical quantities approach a constant. We therefore make the explicit assumption that 
after some retarded time $u=u_{1}, \partial_{u} \phi=0$ on $v=v_{0}$. This assumption has been proven to be very successful [1, [.:[13].

We begin with the first stage of the evolution, i.e., the scattering of the field in the region $u_{0} \leq u \leq u_{1}$. The first two terms in Eq. (6) represent the primary waves in the wave front, while the sum represents backscattered waves. The interpretation of these integral terms as backscatter comes from the fact that they depend on data spread out over a section of the past light cone, while outgoing waves depend only on data at a fixed $u$ [1].

After the passage of the primary waves there is no outgoing radiation for $u>u_{1}$, aside from backscattered waves. This means that $G\left(u_{1}\right)=0$. Hence, for a large $y$ at $u=u_{1}$, the dominant term in Eq. (6) is $\psi\left(u=u_{1}, y\right)=B_{0}(y) G^{(-1)}\left(u_{1}\right)$.

The functions $B_{k}(r)$ satisfy the recursion relation $2 B_{k}^{\prime}-B_{k-1}^{\prime \prime}+V B_{k-1}=0$ for $k>1$, where $B^{\prime} \equiv d B / d y$, and $B_{0}^{\prime}=-V(y) / 2$. Thus, one finds

$$
\psi\left(u=u_{1}, y\right)=\left[M y^{-2} / 2+2 M^{2} y^{-3} \ln y-Q^{2} y^{-3} / 3\right] G^{(-1)}\left(u_{1}\right)[1+O(M / y)] .
$$

This is the dominant backscatter of the primary waves.

With this specification of characteristic data on $u=u_{1}$, we shall next consider the asymptotic evolution of the field. We confine our attention to the region $u>u_{1}, y \gg M,|Q|$. In this region the spacetime is approximated as flat [1.5. (The validity of this approximation is ultimately justified by numerical simulations). Thus, the solution for $\psi$ can be written as

$$
\psi=g^{(0)}(u)+f^{(0)}(v)
$$

Comparing Eq. (8) with the initial data on $u=u_{1}$ Eq. (7), one finds

$$
f(v)=F_{0} v^{-2}+F_{1} v^{-3} \ln v+F_{2} v^{-3},
$$

where

$$
F_{0}=2 M G^{(-1)}\left(u_{1}\right), \quad F_{1}=16 M^{2} G^{(-1)}\left(u_{1}\right), \quad F_{2}=-8 Q^{2} G^{(-1)}\left(u_{1}\right) / 3 .
$$

For late times $t \gg y$ we can expand $g(u)=\sum_{n=0}^{\infty}(-1)^{n} g^{(n)}(t) y^{n} / n$ ! and similarly for $f(v)$. Using these expansions we can rewrite Eq. (8) as 


$$
\psi=\sum_{n=0}^{\infty} K_{0}^{n} y^{n}\left[f^{(n)}(t)+(-1)^{n} g^{(n)}(t)\right],
$$

where the coefficients $K_{0}^{n}$ are those given in [1].

Using the boundary conditions for small $r$ (regularity as $y \rightarrow-\infty$, at the horizon of a black hole, or at $r=0$ for a stellar model), one finds that at late times the terms $h(t) \equiv f(t)+g(t)$ and $f^{(1)}(t)$ must be of the same order (see [5] for additional details). Thus, we conclude that

$$
f(t) \simeq F_{0} t^{-2}+F_{1} t^{-3} \ln t+F_{2} t^{-3}, \quad g(t) \simeq-\left(F_{0} t^{-2}+F_{1} t^{-3} \ln t\right)
$$

and

$$
h(t) \equiv f(t)+g(t)=O\left(M^{2} t^{-3}\right) .
$$

We therefore find that the late-time behaviour of the field for $t \gg y \gg M,|Q|$ is

$$
\begin{aligned}
\psi & \simeq 2 K_{0}^{1} y f^{(1)}(t) \\
& =-2 K_{0}^{1} y\left[2 F_{0} t^{-3}+3 F_{1} t^{-4} \ln (t / M)+3 F_{2} t^{-4}\right][1+O(M / t)] .
\end{aligned}
$$

This is the late-time behaviour of the field at a fixed radius.

\section{NUMERICAL RESULTS}

It is straightforward to integrate Eq. (3) using the method described in [5]. We have used, however, a modified version of the numerical code used in [14], which is essential to achieve the extremely high accuracy needed for the computation (see [14 for additional details).

The late-time evolution of the scalar field is independent of the form of the initial data used. The results presented here are for a Gaussian pulse on $u=0$

$$
\psi(u=0, v)=A \exp \left\{-\left[\left(v-v_{0}\right) / \sigma\right]^{2}\right\},
$$


with a center at $v_{0}=10$ and a width $\sigma=2$. The black-hole mass is set equal to $M=0.5$; this corresponds to the freedom to rescale the coordinates by an overall length scale.

The temporal evolution of the field at a fixed radius $y=50$ is shown in the top panel of Fig. 1. The dominant power-law fall off is manifest at asymptotic late times. In order to study the contamination effect of higher-order terms [see Eq. (14)], we use the notion of a local power index $\gamma$, defined by $\gamma \equiv-t \psi_{t} / \psi$ [0]. Taking cognizance of Eq. (14) we find

$$
\gamma=3+12 \frac{M}{t} \ln (t / M)+O(M / t) .
$$

The approach of the local power index to its well-known asymptotic value $\gamma_{a s y} \rightarrow 3$ is depicts in the bottom panel of Fig. [1. The plot shows that $\gamma \rightarrow 3$ from above, with a qualitative agreement with Eq. (16).

In order to establish quantitatively the physical picture presented in Sec. [II], we define the quantity $\delta \equiv\left(\gamma-\gamma_{\text {asy }}\right)(t / M) / \ln (t / M)$. Figure 2. depicts $\delta$ as a function of $t$ at three surfaces of constant radius $y=5,10$, and 50 (from bottom to top). The numerical result $\delta_{\text {asy }} \simeq 12$ (independently of the value of $y$ ) is in excellent agreement with the analytically predicted behaviour $\delta \rightarrow 12$ [see Eq. (16)]. Thus, Fig. 2. establishes the existence of the contamination term of order $M^{2} t^{-4} \ln (t / M)$. It should be noted that the value of $\gamma_{\text {asy }}$ used in this figure is 2.998 rather than the theoretical value 3. This slight deviation from the theoretical value is expected due to the corrections of order $O(M / t)$ in the expression for $\gamma$ Eq. (16).

\section{SUMMARY AND PHYSICAL IMPLICATIONS}

The purpose of this paper was to derive analytic expressions for the higher-order corrections which "contaminate" the well-known power-law tail in a spherically symmetric gravitational collapse. We have shown, both analytically and numerically, that the dominant correction dies off at late times as $M^{2} t^{-4} \ln (t / M)$. This late-time decay of the contamination is much slower than has been considered so far [9] (see the discussion in Appendix B). 
Aside from being theoretically important, the result Eq. (14) is also of practical importance. It follows that an 'exact' (numerical) determination of the power index demands extremely long integration times. The most accurate method for determining the power index experimentally applies to the concept of the local power index. In this way one discard the relatively large contamination which characterizes the early stages of the evolution. Still, it follows from Eq. (16) that a determination of the power index to within $\sim 1 \%$ requires an integration time of order $t \gtrsim 3000 M$.

The dominant power-law tail is known to be universal in the sense that it is independent of the black-hole parameters [3,5]. We have shown, however, that this universality is removed once we consider higher-order corrections terms - the leading order fingerprint of the blackhole electric charge behaves as $Q^{2} t^{-4}$.

\section{ACKNOWLEDGMENTS}

I thank Tsvi Piran for discussions. This research was supported by a grant from the Israel Science Foundation.

\section{APPENDIX A: LATE-TIME BEHAVIOUR AT NULL INFINITY AND AT THE HORIZON}

It follows from Eqs. (8), (9), and (12) that the asymptotic behaviour of the field at future null infinity $s c r i_{+}$(i.e., at $v \gg u$ ) is

$$
\psi(v \gg u, u) \simeq g^{(0)}(u) \simeq-\left(F_{0} u^{-2}+F_{1} u^{-3} \ln u\right)
$$

We finally consider the behaviour of the field at the black-hole outer horizon $r_{+}$. As $y \rightarrow-\infty$ the curvature potential Eq. (4) is exponentially small, and the general solution to Eq. (3) can be written as $\psi=\alpha(u)+\gamma(v)$. On $v=v_{0}$ we take $\partial_{u} \phi=0$ (for $\left.u \rightarrow \infty\right)$. Thus, $\alpha(u)$ must be a constant, and with no loss of generality we can choose it to be zero. We next expand $\gamma(v)$ for $t \gg|y|$ as 


$$
\psi=\gamma(v)=\sum_{n=0}^{\infty} \frac{1}{n !} \gamma^{(n)}(t) y^{n} .
$$

In order to match the $y \ll-M$ solution Eq. (A2) with the $y \gg M$ solution Eq. (14), we make the ansatz $\psi \simeq \psi_{\text {stat }}(y)\left[2 F_{0} t^{-3}+3 F_{1} t^{-4} \ln (t / M)+3 F_{2} t^{-4}\right]$ for the solution in the region $y \ll-M$ and $t \gg|y|$. In other words, we assume that the solution in the $y \ll-M$ region has the same late time $t$ dependence as the $y \gg M$ solution. This assumption has been proven to be very successful for the leading order behaviour of both neutral [5] and charged 113] fields. Using this assumption, one finds $\gamma(t)=\Gamma_{0}\left[2 F_{0} t^{-3}+3 F_{1} t^{-4} \ln (t / M)+3 F_{2} t^{-4}\right]$, where $\Gamma_{0}$ is a constant. Thus, the asymptotic behaviour of the field at the black-hole horizon is

$$
\psi(u \rightarrow \infty, v)=\Gamma_{0}\left[2 F_{0} v^{-3}+3 F_{1} v^{-4} \ln (v / M)+3 F_{2} v^{-4}\right]
$$

\section{APPENDIX B: A NOTE ON ANDERSSON'S ANALYSIS}

The first attempt to calculate higher-order corrections to the dominant power-law tail was made by Andersson [9] (in the context of the Schwarzschild spacetime). This analysis was based on an approximated curvature potential in the region far away from the black hole [see Eq. (26) of [9]]. While this approximated potential simplifies the analysis, it actually misses the genuine leading order corrections terms. The leading order correction in [9] was found to be of order $M t^{-5}$. This (artificial) result is caused by the fact that terms of order $M / r^{3}$ (and smaller) were totally neglected by the approximated curvature potential used in [9].

Moreover, the approximated approach presented in [9], if extended along the same lines to a Reissner-Nordström spacetime would imply that the influence of the black-hole electric charge on the late-time tail vanishes identically (i.e., it would vanish to any order in $t^{-1}$ ). This result is again a direct consequence of the fact that the approximated approach of [9] does not take into account curvature terms of order $Q^{2} / r^{4}$ (and $M^{2} / r^{4}$ ) which appear in the (exact) curvature potential. 


\section{REFERENCES}

[1] R.H. Price, Phys. Rev. D5, 2419 (1972).

[2] K.S. Thorne, p. 231 in Magic without magic: John Archibald Wheeler Ed: J.Klauder (W.H. Freeman, San Francisco 1972).

[3] J. Bičák, Gen. Relativ. Gravitation 3, 331 (1972).

[4] E.W. Leaver, Phys. Rev. D34, 384 (1986).

[5] C. Gundlach, R.H. Price, and J. Pullin, Phys. Rev. D 49, 883 (1994).

[6] C. Gundlach, R.H. Price, and J. Pullin, Phys. Rev. D 49, 890 (1994).

[7] L. M. Burko and A. Ori, Phys. Rev. D 56, 7820 (1997).

[8] E. S. C. Ching, P. T. Leung, W. M. Suen, and K. Young, Phys. Rev. D 52, 2118 (1995); Phys. Rev. Lett. 74, 2414 (1995).

[9] N. Andersson, Phys. Rev. D 55, 468 (1997).

[10] L. Barack, Phys. Rev. D 59, 044017 (1999).

[11] Y. Sun and R. H. Price, Phys. Rev. D 38, 1040 (1988).

[12] N. Andersson, Phys. Rev. D 51, 353 (1995).

[13] S. Hod and T. Piran, Phys. Rev. D 58, 024017 (1998).

[14] S. Hod and T. Piran, Phys. Rev. D 55, 3485 (1997). 


\section{FIGURES}
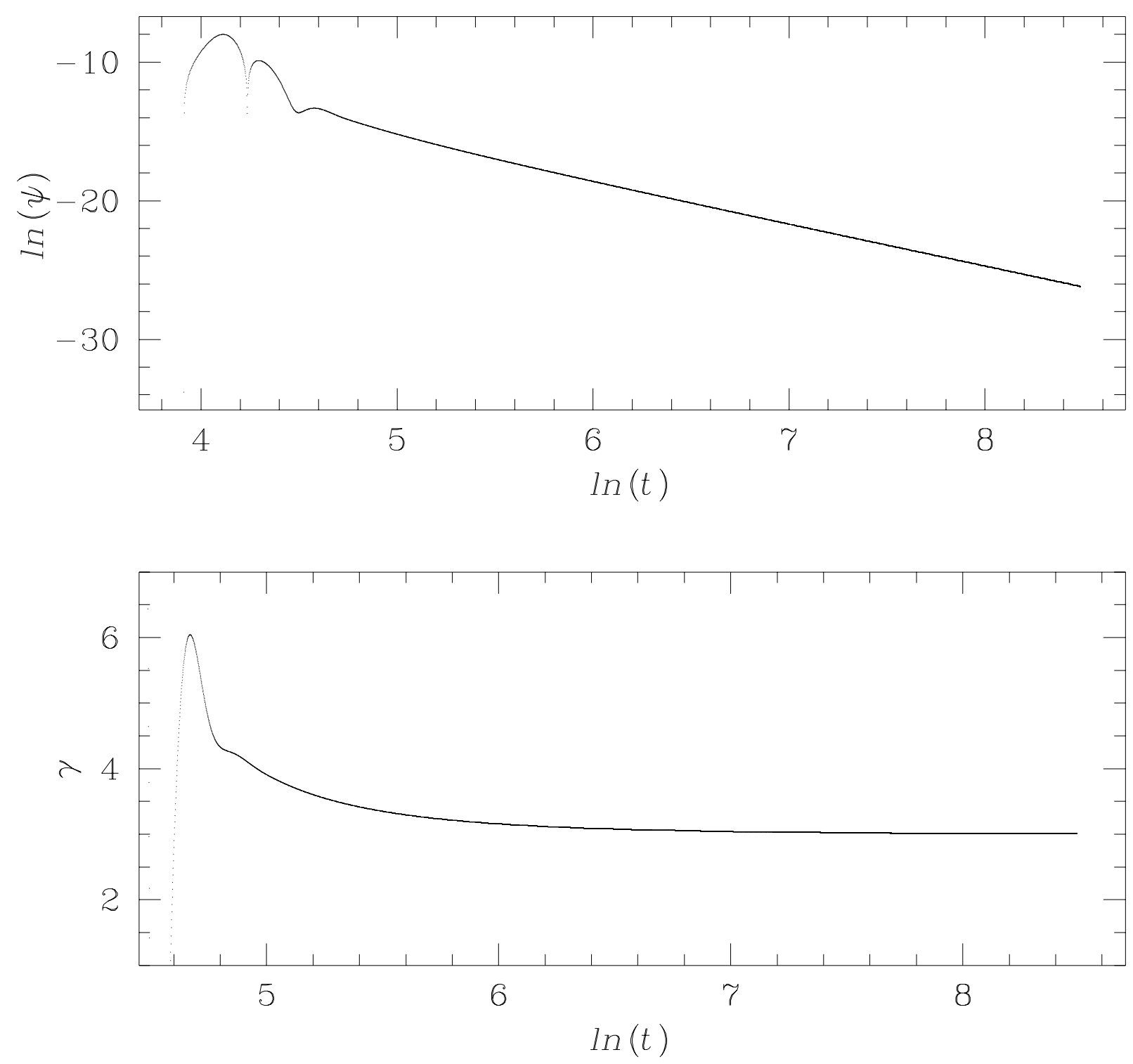

FIG. 1. Temporal evolution of the scalar field, evaluated at $y=50$ in a Schwarzschild spacetime with $M=0.5$. The initial data is a Gaussian distribution with $v_{0}=10$ and $\sigma=2$. The asymptotic power-law fall off is manifest at late times (top panel). The bottom panel depicts the evolution of the local power index $\gamma \equiv-t \psi_{t} / \psi$. The power index approaches the well-known asymptotic value $\gamma_{a s y}=3$ at late times. 


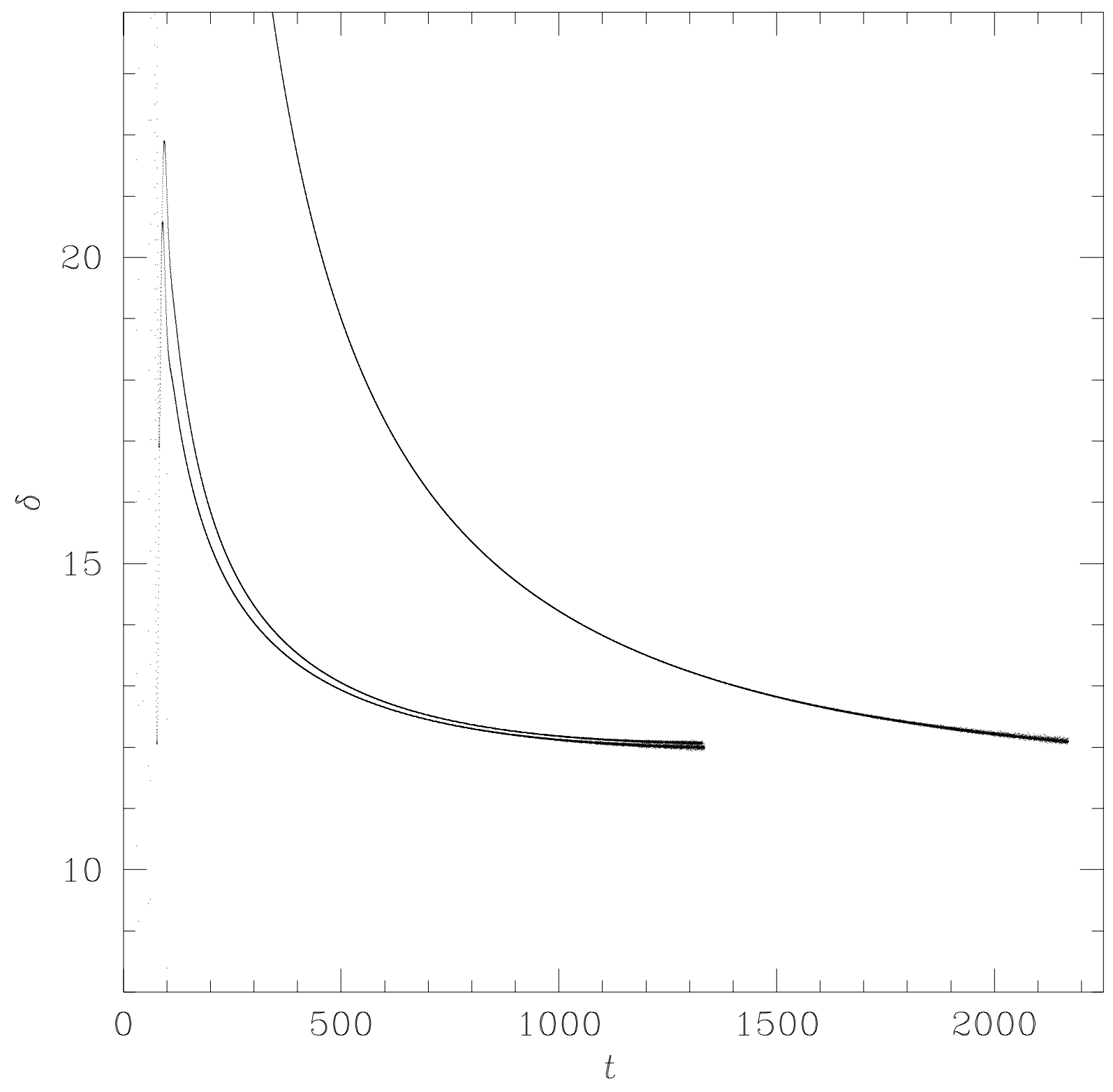

FIG. 2. The time evolution of the quantity $\delta \equiv\left(\gamma-\gamma_{a s y}\right)(t / M) / \ln (t / M)$, evaluated at $y=5,10$, and 50 (from bottom to top). The asymptotic value $\delta_{a s y} \simeq 12$ is in excellent agreement with the analytically predicted behaviour $\delta \rightarrow 12$ at late times. This result establishes the existence of the contamination term of order $M^{2} t^{-4} \ln (t / M)$. 